\title{
ANALYSIS OF SUB-GRID MODELING EFFECTS IN THE SIMULATION OF THE SINGLE-PHASE TURBULENT FLOW IN AN INDUSTRIAL CYCLONE SEPARATOR
}

\begin{abstract}
R. V. Salvo,
F. J. Souza,

and D. A. M. Martins

Universidade Federal de Uberlândia - UFU Faculdade de Engenharia Mecânica - FEMEC

Av. João Naves de Ávila, 2121,

Bairro Santa Mônica

38408-144, Uberlândia, Minas Gerais, Brasil rvsalvo@hotmail.com

ABSTRACT

In the present work two turbulence modeling approaches, namely Large Eddy Simulation and Detached Eddy Simulation, are employed to predict turbulent, swirling flow within an industrial cyclone separator running at Reynolds number 267,000. The results from three LES models, Smagorinsky, dynamic and Yakhot, and the SST-DES model of Strelets have been compared to experimental results for the average axial and tangential velocities. The Navier-Stokes solver is based on an unstructured, finite volume, cell-centered algorithm such that the details of the geometry can be accurately represented. Based on the comparison with the experimental results, it has been found that the Yakhot model provides the most accurate predictions for the tangential velocities, whereas the dynamic LES and the Smagorinsky models overpredict it and the SST-DES model underpredicts it. However, the conclusions are different regarding the axial velocity. Implications of the turbulence modeling for the particle separation are discussed.
\end{abstract}

Keywords: cyclone separator, les, des, dynamic les model

\section{NOMENCLATURE}

$\mathrm{A}^{+} \quad$ van Driest constant

c square of $\mathrm{Cs}$

C Yakhot turbulence model constant

$\mathrm{C}_{\text {DES }}$ SST-DES constant

Cs Smagorinsky turbulence model constant

$\mathrm{F}_{\text {DES }} \quad$ SST-DES turbulence lengthscale, $\mathrm{m}$

$\mathrm{F}_{1} \quad$ blending function

$\mathrm{F}_{2} \quad$ blending function

$\mathrm{G}$ filtering function based on grid spacing

$\mathrm{H}$ Heavyside Ramp function

$\mathrm{L}_{\mathrm{ij}} \quad$ Global Leonard tensor, $\mathrm{m}^{2} / \mathrm{s}^{2}$

$\mathrm{L}_{\mathrm{kw}} \quad$ turbulence lengthscale, $\mathrm{m}$

$\mathrm{M}_{\mathrm{ij}}$ Tensor (dynamic turbulence model), $\mathrm{m}^{2} / \mathrm{s}^{2}$

$\bar{p}^{*}$ modified filtered pressure, $\mathrm{Pa}$

$\bar{S} \quad$ filtered strain rate, $1 / \mathrm{s}$

$\mathrm{y}^{+} \quad$ distance to nearest wall, in wall units

\section{Greek symbols}

$\Delta \quad$ grid spacing, $\mathrm{m}$

$\kappa \quad$ turbulence kinetic energy, $\mathrm{m}^{2} / \mathrm{s}^{2}$

$\mu \quad$ dynamic viscosity, $\mathrm{kg} / \mathrm{m} . \mathrm{s}$

$\rho$ density, $\mathrm{kg} / \mathrm{m}^{3}$

$\sigma \quad$ SST-DES turbulence model constant

$\omega \quad$ specific turbulent dissipation ratio, $1 / \mathrm{s}$

\section{Subscripts}

sgs sub-grid

t turbulent tot total

\section{Superscripts \\ - $\quad$ grid filter process \\ $\sim \quad$ test filter process}

\section{INTRODUCTION}

Cyclone separators have been used for over a century, during this period, although the design of these devices has undergone considerable changes, their operating principles have not changed. The separation process in these devices occurs due to the strong swirling motion of the flow, which causes the action of a centrifugal force. This force promotes the phase separation. Predicting the separation efficiency of cyclone involves predicting how particles behave in the separation space. In order to do this, it is necessary to know the velocity distribution of the gas, which is the most basic step possible into the correct prediction of the separation efficiency in cyclone separators. Unfortunately, the flow field in the interior of such devices is extremely complex, making the correct gas flow prediction an oft-sought goal but seldom achieved in practice (Hoffman and Stein, 2008). It has been exhaustively demonstrated, in the literature, that numerical simulation based on conservation equations provides considerable insight into these complicated flows. Indeed, CFD simulations are commonly employed in an industrial environment for predicting cyclone performance. 
It is also know from literature that traditional RANS models do not apply in a proper manner to CFD simulations of strongly swirling flows and even more sophisticated models like the RSM face some difficulties in this type of flow (Slack et al. (2000); Wegner et al. (2004); Bernardo (2005); Narasimha et al. (2006); Narashimha et al. (2007); Shalaby (2007); Gronald and Derksen (2010); just to name a few). Based on the literature survey and in the authors believe in the LES methodology the present work is based on Large Eddy Simulation for turbulence modeling. Thus a comparative study between the results obtained with three different LES turbulence models and a DES turbulence model in the simulation of a cylinder on cone cyclone separator operating at Reynolds number 267,000 was performed.

The results shown consistent agreement with experimental data and complementary information about the complex flow field in the interior of the device, such some secondary flows, could be properly investigated.

\section{MATHEMATICAL FORMULATION}

The conservation of mass and the Navier-Stokes equations for a general incompressible, Newtonian flow can be written, adopting the Einstein convention, respectively as:

$$
\begin{gathered}
\frac{\partial \rho u_{i}}{\partial x_{i}}=0 \\
\frac{\partial \rho u_{i}}{\partial t}+\frac{\partial}{\partial x_{j}}\left(\rho u_{i} u_{j}\right)= \\
-\frac{\partial p}{\partial x_{i}}+\frac{\partial}{\partial x_{j}}\left[\mu\left(\frac{\partial u_{i}}{\partial x_{j}}+\frac{\partial u_{j}}{\partial x_{i}}\right)\right] \\
f(\vec{x}, t)=\bar{f}(\vec{x}, t)+f^{\prime}(\vec{x}, t)
\end{gathered}
$$

Applying a filtering process to the above equations, it is possible to separate the larger scales of motion, which are related to the lowest frequencies, from the smallest scales, which are related to the higher frequencies. Considering a spatial filtering process, it can be defined as shown in Eq. (3).

Where the filtered part is given by:

$$
\bar{f}(\vec{x}, t)=\int_{D} f\left(\vec{x}^{\prime}, t\right) G\left(\vec{x}-\vec{x}^{\prime}\right) d \vec{x}^{\prime}
$$

And the filtering function can be defined as:

$$
G(\vec{x})=\frac{1}{\Delta_{x} \Delta_{y} \Delta_{z}}, i f|\vec{x}| \leq \frac{\Delta}{2}
$$

Or zero otherwise.

Applying the filtering processes defined above in Eq. (2), results in:

$$
\begin{aligned}
& \frac{\partial \rho \bar{u}_{i}}{\partial t}+\frac{\partial}{\partial x_{j}}\left(\rho \bar{u}_{i} \bar{u}_{j}\right)= \\
& \quad-\frac{\partial \bar{p}^{*}}{\partial x_{i}}+\frac{\partial}{\partial x_{j}}\left[\left(\mu+\mu_{t}\right)\left(\frac{\partial \bar{u}_{i}}{\partial x_{j}}+\frac{\partial \bar{u}_{j}}{\partial x_{i}}\right)\right]
\end{aligned}
$$

In Eq. (6), the over-bar denotes a filtered quantity, the asterisk denotes that the pressure is actually modified, and includes the Turbulence Kinetic Energy and $\mu_{t}$ is the turbulent viscosity (this term represents the energy dissipation present on the smallest scales of the flow, which are not resolved in LES, so it has to be modeled).

\section{TURBULENCE MODELS}

In the present work three different LES turbulence models were used, and a small description of each is given bellow.

\section{Smagorinsky sub-grid turbulence model}

This is the earliest and simplest LES model. It was developed by Smagorinsky in 1963 for weather forecasts (Ferziger and Peric, 2002). In this model the turbulent viscosity can be calculated as follows:

$$
\mu_{t}=\rho\left(C \Delta^{2}\right) \bar{S}
$$

where:

$$
\bar{S}=\sqrt{\bar{S}_{i j} \bar{S}_{i j}}, \text { and } \bar{S}_{i j}=\frac{1}{2}\left(\frac{\partial \bar{u}_{i}}{\partial x_{j}}+\frac{\partial \bar{u}_{j}}{\partial x_{i}}\right)
$$

In Eq. (7) Cs is a parameter to be determined, as it varies from flow to flow and from one region in a flow field to another. In the present work the value 0.1 was adopted. Since in this model the eddy viscosity is not damped in near wall regions as it should, in this work the Smagorinsky model is utilized in conjunction with the van Driest wall damping function, which is given by:

$$
C s=C s_{0}\left(1-e^{-y+/ A+}\right)^{2}
$$

Where $\mathrm{y}^{+}$is the distance to the nearest wall in wall units and $\mathrm{A}^{+}$is a constant $(25$, in the present work). 
For more information about this model the interest reader is referred to the book by Ferziger and Peric (2002).

\section{Dynamic sub-grid turbulence model}

Although the Smagorinsky sub-grid model can be, and it has been, used in a great variety of flows presenting really good results, it has some drawbacks, for instance, the Cs parameter is not constant, and it may vary drastically from one type of flow to another. Thus, in some cases it may require a precalibration, which is time consuming. Also, it requires additional formulation to provide the necessary damping in the calculated eddy viscosity for near wall flows (for example, the van Driest damping function).

The dynamic sub-grid model attempts to overcome these deficiencies by locally calculating the eddy viscosity coefficient to reflect closely the state of the flow. This is done by sampling the smallest resolved scales and using this information to model the sub-grid scales (Germano et al., 1990). According to Silveira-Neto (2002), two different filters are utilized:

- The grid filter, in which the grid dimensions are used to calculate its characteristic length.

- The test filter, in which a multiple of grid size, normally two, is used to calculate the characteristic length.

A brief summary of the formulation used in this model for incompressible flows, with the modifications proposed by Lilly (1991), is presented below:

$$
C=-\frac{1}{2} \frac{L_{i j} M_{i j}}{M_{i j} M_{i j}}
$$

where:

$$
M_{i j}=\widetilde{\bar{\Delta}}^{2}|\widetilde{\bar{S}}| \widetilde{\bar{S}}_{i j}-\bar{\Delta}^{2}|\bar{S}| \bar{S}_{i j}
$$

and:

$$
L_{i j}={\overline{u_{i} u_{j}}}_{-} \widetilde{\bar{u}}_{i} \widetilde{\bar{u}}_{j}
$$

In the above equations the over bar denotes the grid filter process while the over tilde denotes the test filter process.

The model parameter produced by Eq. (10) is a rapidly varying function of spatial coordinates and time so the eddy viscosity may take large values of both signs. This can and does lead to numerical divergence, so a usual technique is clip the negative turbulence viscosity. For more information about this model the interested reader is referred to the original paper presented by Germano (Germano et al, 1990) and the work of Lilly (1991).

\section{Yakhot RNG sub-grid turbulence model (Yakhot et al., 1986)}

The main difference between this model and the Smagorinsky model is in the way that total viscosity is calculated:

$$
\mu_{t o t}=\mu\left[1+H\left(\frac{\mu_{s g s}^{2} \mu_{t o t}}{3}-C\right)\right]^{1 / 3}
$$

In Eq. (13) the sub-grid viscosity is calculated in the same way as in the standard Smagorinsky model, Eq. (7), however in this model $\mathrm{Cs}$ is a theoretical constant $(\mathrm{Cs}=0.157)$. Also in Eq. (13) $\mathrm{C}$ is another constant $(\mathrm{C}=100)$ and $\mathrm{H}$ is the Heaviside Ramp function, which yields zero if the argument assume negative values. This model correctly yields zero subgrid viscosity in low Reynolds number flows. For more information about this model the interested reader is referred to the original work of Yakhot (Yakhot et al., 1986) and the paper by Slack et al (2000).

\section{SST-DES turbulence model}

A DES (Detached Eddy Simulation) model behaves, in a general way, as a RANS turbulence model in near wall regions and, as the distance to the wall increases, it gradually turns into a LES model. The main idea used behind this model is that the structures present in near wall regions should be provided by the RANS model and not calculated. The merge of the RANS and LES models normally occurs in the inner boundary layer. Thus, in the region where the LES model is used, it is also possible to use a coarser numerical grid, once, at least from a theoretical point of view, the grid spacing in this region is dictated by the necessity to resolve the larger turbulent scales of the flow field.

The DES methodology can be applied to any RANS model. However, this requires that the characteristic turbulent length of the base model be modified in an appropriate manner. Considering this, an interesting alternative is to use the DES methodology with the RANS SST model from Menter (1992). The application of the SST-DES consists only in the modification of the destruction term in the kinetic turbulence equation from the original SST model:

$$
\frac{\partial \rho \kappa}{\partial t}+\frac{\partial \rho \bar{u}_{j} \kappa}{\partial x_{i}}=
$$




$$
P_{\kappa}-\beta^{*} \rho \kappa \omega F_{D E S}+\frac{\partial}{\partial x_{j}}\left[\left(\mu+\sigma_{\kappa} \mu_{t}\right) \frac{\partial \kappa}{\partial x_{j}}\right]
$$

In which:

$$
F_{D E S}=\max \left[\frac{L_{\kappa \omega}}{C_{D E S} \Delta}\left(1-F_{2}\right), 1\right]
$$

$\mathrm{L}_{\mathrm{kw}}$ is the turbulence lengthscale. Like the other constants in the SST model, $\mathrm{C}_{\mathrm{DES}}$ is blended using the F1 function. $\Delta$ is the mesh characteristic lengthscale, computed as the largest element edge in this work. For more details, the reader is referred to the original work by Strelets (2001).

\section{NUMERICAL METHOD}

\section{Numerical Code}

For the simulations, the code UNSCYFL3D (Unsteady Cyclone Flow - 3D), was used. This inhouse code is being developed as a dedicated tool for simulating highly rotational flows, aiming at cyclones/hydrocyclones separators and swirl tubes. It is based on a cell-centered, finite volume method on unstructured grids and is thus able to calculate flows within and over complex geometries. Three advective schemes are available: second-order centered (CDS), first and second-order upwind. For the timeadvancement, the implicit first and second-order Euler schemes can be blended. The solver is segregated and velocity and pressure are coupled by the SIMPLE (Semi-Implicit Method for PressureLinked Equations). The non-smoothness of the grid and non-orthogonality effects are also taken into account (Ferziger and Peric, 2002). For the solution of the linear systems the biconjugate gradient (Ferziger and Peric, 2002) and the algebraic multigrid (Notay, 2008) methods were used. In all the simulations, the centered scheme was used for the momentum equations, whereas the first order upwind was used in the turbulence transport equations. The time-advancement was also second-order.

\section{Numerical Procedure}

The cyclone geometry simulated is a high efficiency Stairmand cyclone, as depicted in Fig. 1. The cylinder diameter is $0.205 \mathrm{~m}$. This is the same geometry experimentally studied by Boysan et al. (1983) and numerically simulated by Ayres et al. (1983), Slack et al. (2000) and Dias et al. (2008). In all the simulations performed in this work, the air properties were: density $1.225 \mathrm{Kg} / \mathrm{m}^{3}$ and dynamic viscosity $1.7894 \mathrm{E}-05 \mathrm{Kg} / \mathrm{m} . \mathrm{s}$. The boundary conditions adopted in the simulations were:
- At the inlet a normal, uniform velocity profile of $19.03 \mathrm{~m} / \mathrm{s}$, yielding a Reynolds number of 267,000 , was used.

- At the overflow outlet the pressure was prescribed.

- All the cyclone walls were considered as no-slip walls and the cone apex was also considered as a wall, since only the gas phase was simulated (closed bottom cyclone).

In this work the wall thickness of the vortex finder was considered as D/40, since no reference for this thickness was found.

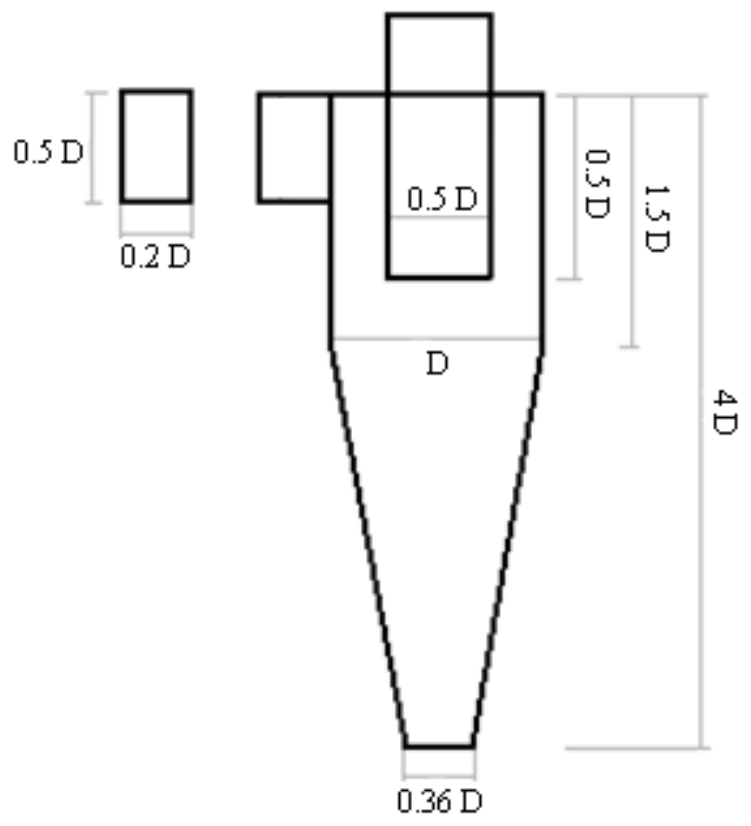

Figure 1. Cyclone geometry.

Initially, a steady state case was run with the SST turbulence model, using a first order upwind scheme for momentum and continuity equations and a convergence criterion of $1.0 \mathrm{E}-05$ for the momentum and continuity residuals. Then the transient simulations were performed using CDS scheme for the conservation equations and a time step of 2.0E-05 $\mathrm{s}$, with a convergence criteria of $1.0 \mathrm{E}-04$. The difference in the convergence criteria used in the steady state and transient cases its due to some previous studies which showed that a tighter convergence criterion in the steady state case results in a much faster transient simulation, since fewer iterations per time step are required.

The average residence time for this cyclone is $0.25 \mathrm{~s}$. Based on this residence time, the total physical time simulated was $1.0 \mathrm{~s}$. From these, the first quarter was discarded and the average was sampled with the remaining $0.75 \mathrm{~s}$. 

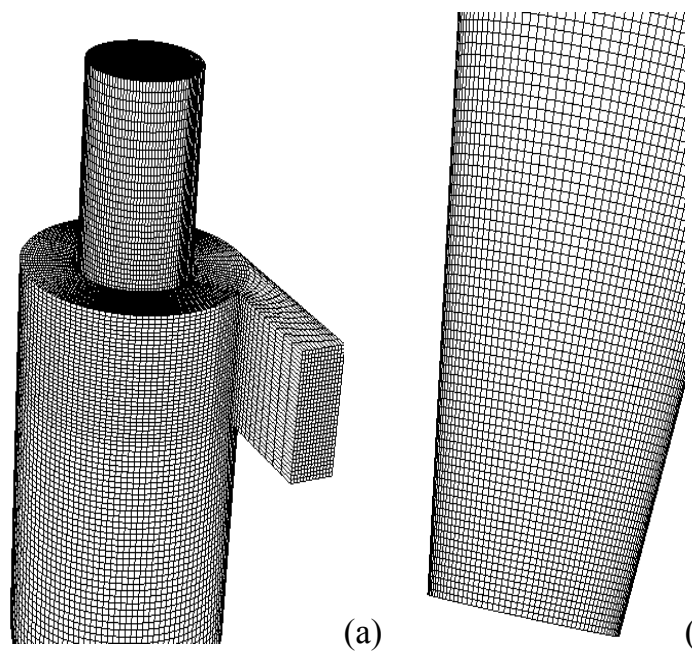

(b)

Figure 2. Unstructured numerical grid with approximately 1,020,000 elements; (a) refinement at the top; (b) refinement at the bottom.

\section{RESULTS}

\section{Comparison with Experimental Data}

In this section a quantitative comparison between numerical results obtained with the different turbulence models and the available experimental data will be shown. The comparison is restricted to the analysis of radial profiles of mean tangential and axial velocities at seven different positions inside the cyclone separator, three in the cylindrical section and four in the conical section. All the profiles were retrieved from plane $\mathrm{x}=0$ (slice in the central position of the cyclone, perpendicular to the cyclone inlet) and the axial position is measured from the cyclone bottom (cone apex) to the top.

Figures 3, 4 and 5 show the experimental averaged values for the tangential and axial velocities, along with the corresponding simulation results for the cylindrical part of the cyclone (positions $\mathrm{Y}=0.65 \mathrm{~m} ; \mathrm{Y}=0.59 \mathrm{~m} ; \mathrm{Y}=0.56 \mathrm{~m}$, respectively). The agreement regarding the experimental mean tangential velocity is particularly good for the Yakhot turbulence model, as indicated by the tangential velocity peak, although in section $\mathrm{Y}=0.65$ (Fig. 3) it is slightly dislocated to the right. The other three models performed reasonably well in this section, although the dynamic and Smagorinsky models overpredicted the maximum velocity, and the DES model under-predicts it. Regarding the averaged axial velocity profiles, the results obtained with the standard Smagorinsky model and with the dynamic model are pretty close to the experimental ones, closely followed by the results obtained with the Yakhot model. The DES model was not able to capture the velocity valley near the cyclone center, and therefore completely missed the axial velocity profile in that region.

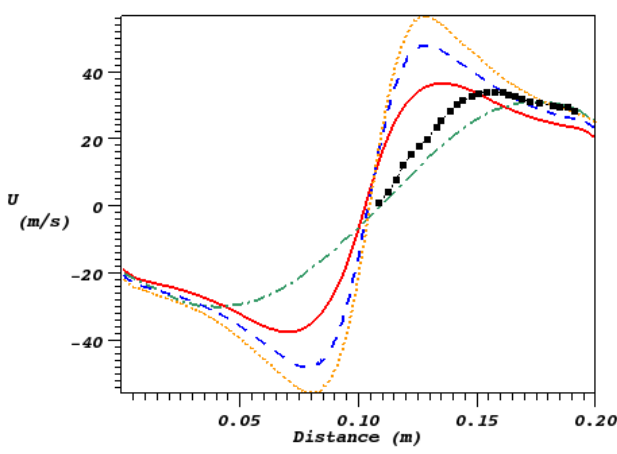

(a)

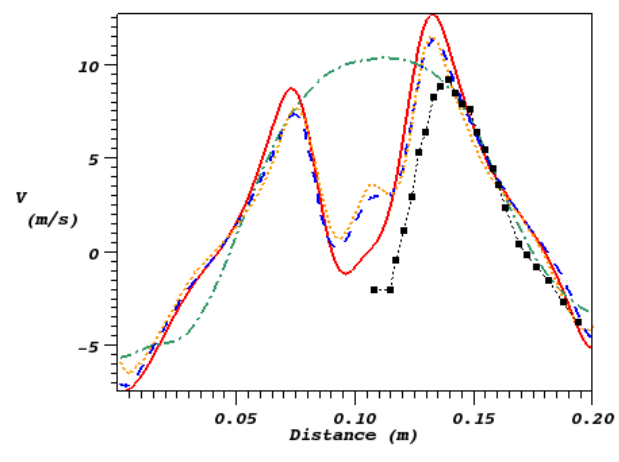

(b)

Figure 3. Radial profiles of average tangential (a), and axial (b) velocities at the axial position 0.65

m. — Yakhot RNG sub-grid model, - - - standard Smagorinsky, Dynamic sub-grid model, $\ldots$ SSTDES model; .......experimental data.

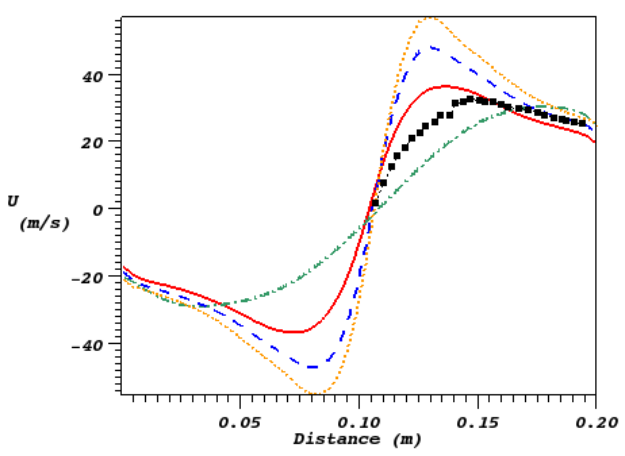

(a)

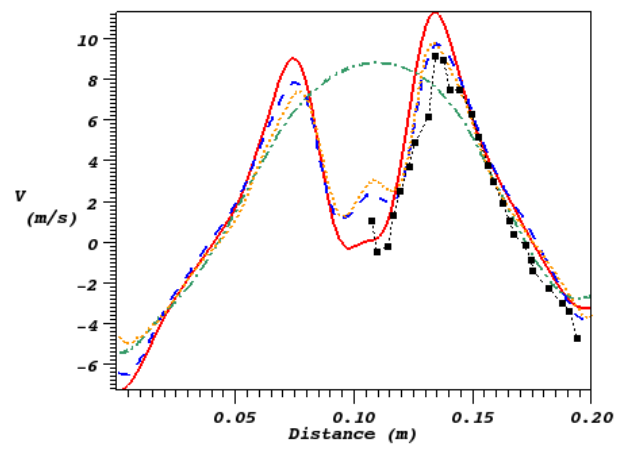

(b)

Figure 4. Radial profiles of average tangential (a), and axial (b) velocities at the axial position 0.59 m. See Fig. 3 for caption. 

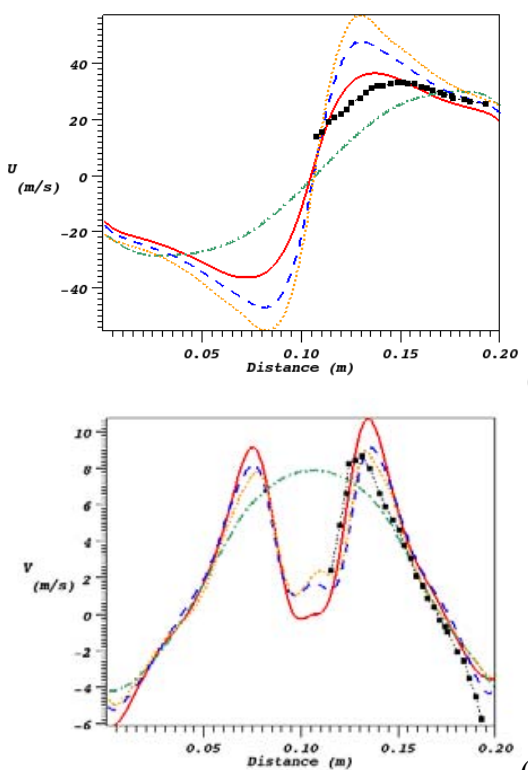

(a)

(b)

Figure 5. Radial profiles of average tangential (a), and axial (b) velocities at the axial position 0.56 m. See Fig. 3 for caption.

Figures 6, 7, 8 and 9 show the numerical and experimental averaged tangential and axial velocities, along the cyclone conical section in the axial positions $0.38 \mathrm{~m}, 0.35 \mathrm{~m}, 0.20 \mathrm{~m}, 0.17 \mathrm{~m}$ respectively. Basically the same trend obtained in the cylindrical section is maintained in the conical section. The main differences are the small radial displacements of the numerical axial velocity yielded by the Smagorinsky, the dynamic and the Yakhot RNG turbulence models at sections $0.38 \mathrm{~m}, 0.35 \mathrm{~m}$ and $0.20 \mathrm{~m}$.

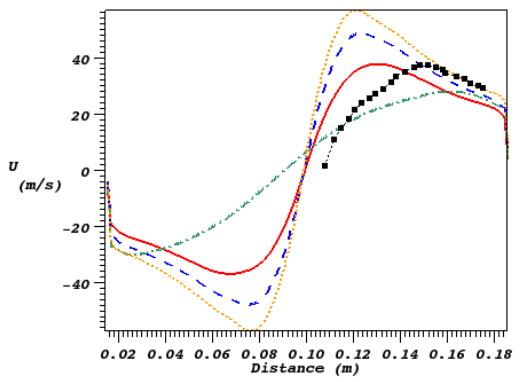

(a)

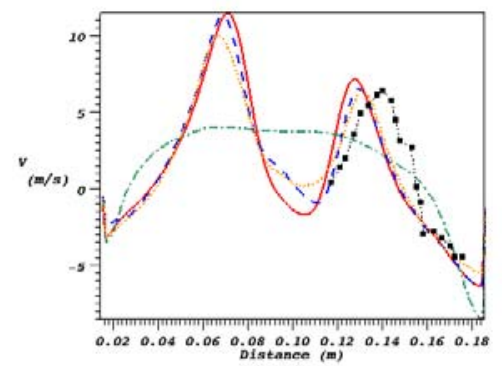

(b)

Figure 6. Radial profiles of average tangential (a), and axial (b) velocities at the axial position $0.38 \mathrm{~m}$. See Fig. 3 for caption.
One interesting feature is generated from the SST-DES turbulence model in sections $0.20 \mathrm{~m}$ and $0.17 \mathrm{~m}$. It displays a small valley for the axial velocity profile in the central region, but does not seem to be capable of capturing the Rankine vortex, when the tangential velocity profile is analyzed. This is probably due to the fact that in this model the transition RANS-LES is based on the grid length scale. Although the axial length of the elements is smaller in this region (near the bottom, Fig. 2(b)) when compared to the elements near the cyclone center, Fig. 2 (a), a much finer grid would be necessary to correctly capture the velocity profiles with this model. The original idea of the DES models was to reduce the cost of an LES by not solving the turbulence lengthscales in the near-wall region, i.e., switching to the RANS mode. As a consequence, if high-aspect ratio elements exist in the core flow, the LES mode will be inhibited, which will in turn reduce the precision of the swirling flow calculation.

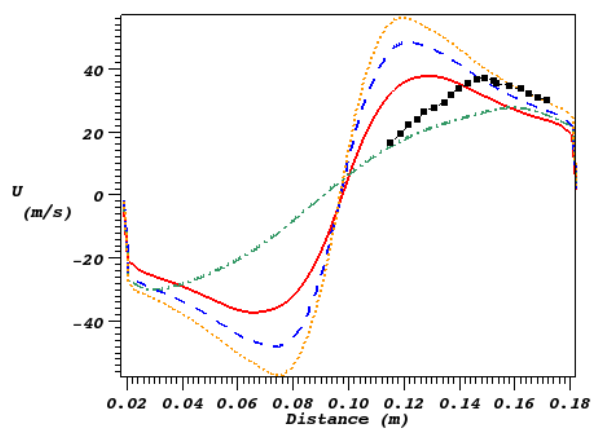

(a)

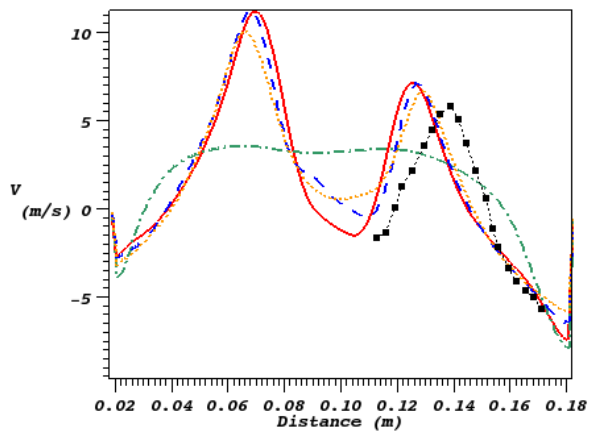

(b)

Figure 7. Radial profiles of average tangential (a), and axial (b) velocities at the axial position 0.35 m. See Fig. 3 for caption.

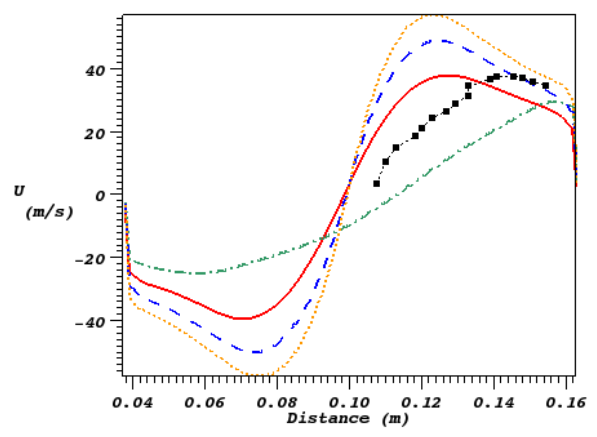

(a) 


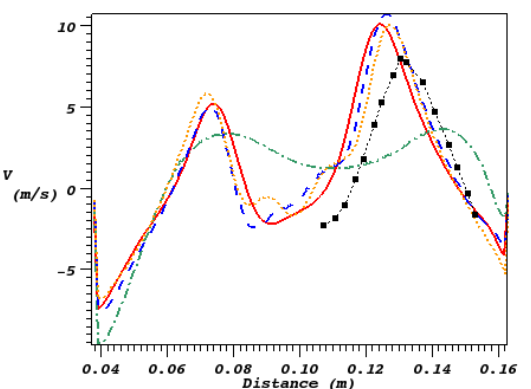

(b)

Figure 8 . Radial profiles of average tangential (a), and axial (b) velocities at the axial position 0.20 m. See Fig. 3 for caption.
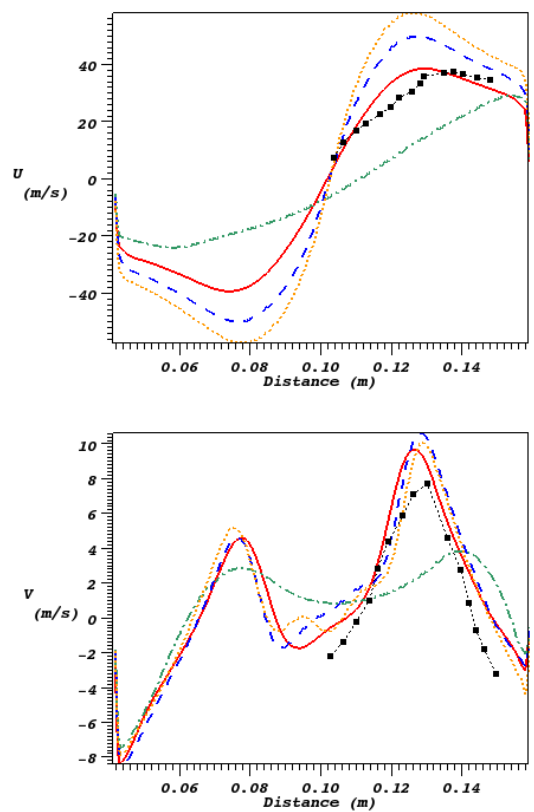

(a)

Figure 9. Radial profiles of average tangential (a), and axial (b) velocities at the axial position 0.17 m. See Fig. 3 for caption.

\section{Secondary Flow Phenomena and the Effect of Turbulence Models}

Figure 10 (a) shows several streamlines for the averaged flow field obtained with the Yakhot RNG turbulence model. It can be noticed from this figure that some streamlines go all the way from the inlet duct to the cyclone bottom, reverse their axial movement, and then go from the bottom to the overflow duct. Other streamlines do not even reach half way down in the cyclone body. This is a consequence of the fluid particle positions inside the inlet duct, and this effect can be seen in more detail in Figs. 10 (b)-(f).

Figures 10 (b) and (c) show that streamlines traced from the lower section of the inlet duct reach the cyclone bottom regardless the radial position in the inlet. Figs. 10 (d) and (e) show that fluid particles injected in the upper inlet duct section have a great dependence upon the radial position of injection (in Fig. 10 (d) the streamline reaches the conical section, while in Fig. 10 (e) it goes to the cyclone top, spins around the vortex finer and then enters it, showing almost zero separation effect (typical short-circuit flow). Fig. 10 (f) show a streamline released in the middle of the inlet duct.

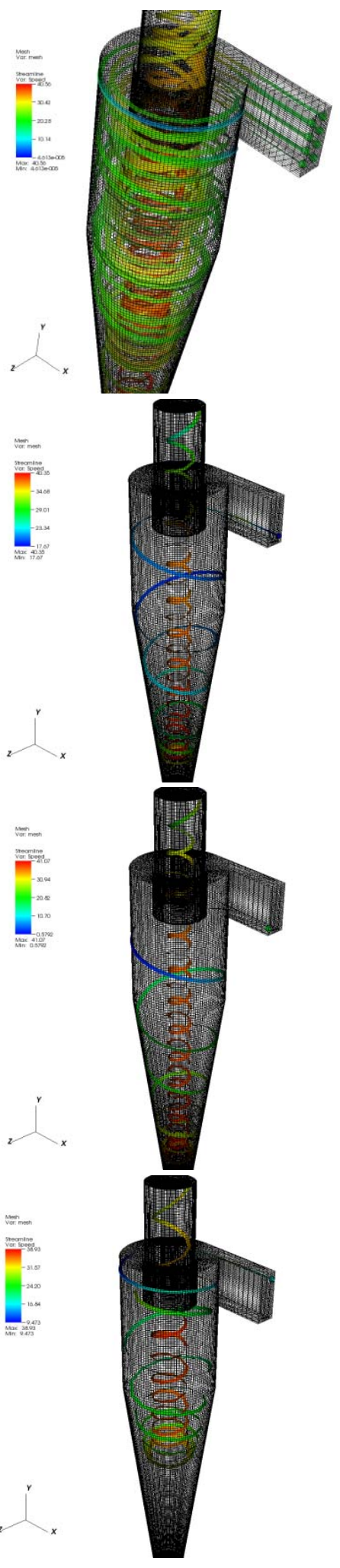

(a)

(b)

(c) 


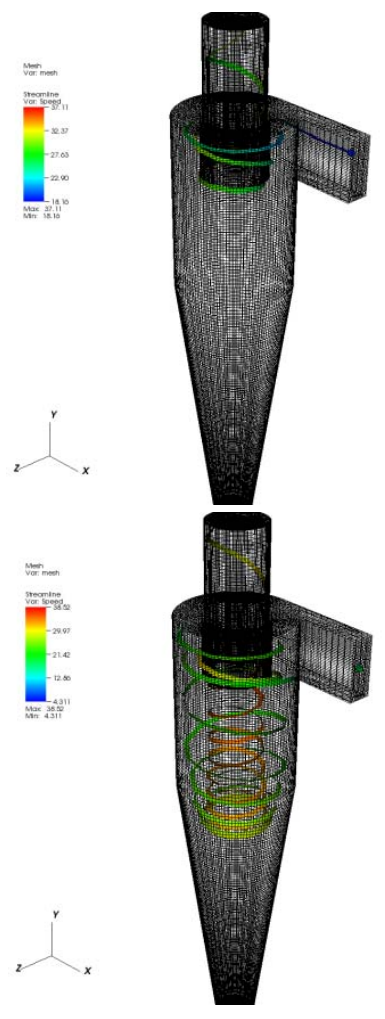

(e)

Figure 10. Streamlines as a function of inlet position for the average vector velocity field (results for the Yakhot LES model).

Figure 11 shows the effect of the turbulence modeling on the streamlines. Streamlines released in the same position are shown for the different turbulence models employed.

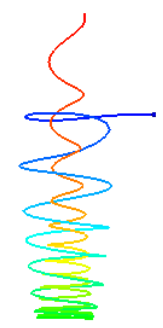

(a)

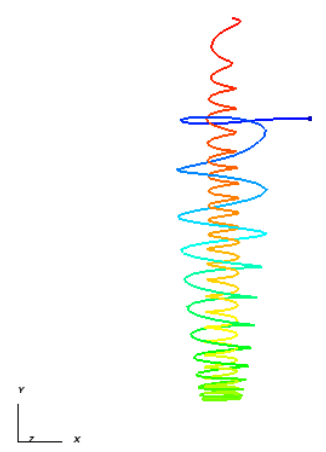

(b)

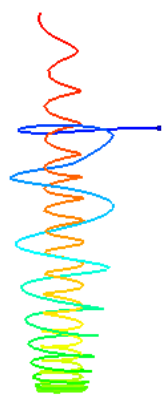

(c)

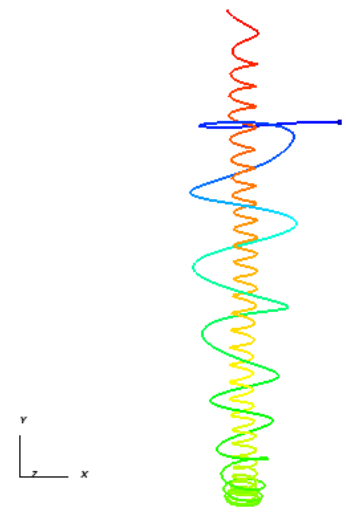

(d)

Figure 11. Streamlines from averaged flow fields obtained with different turbulence models ( $\mathrm{a}-$ SST-DES; b - Dynamic; c - Smagorinsky; d Yakhot).

It is interesting to note in the figure above that in the SST-DES simulation the streamline is shorter, barely reaching the cyclone conical section, followed by the streamlines in the Smagorinsky and Dynamic model, respectively. This is somewhat expected, since the maximum tangential velocity grows from the DES to the Dynamic LES turbulence model. On the other hand, the maximum tangential velocity obtained with the Yakhot model is an intermediate value between the SST-DES and the Smagorinsky model, and it has showed the longest streamline, which goes all the way to the cyclone bottom.

\section{CONCLUSIONS}

Large Eddy Simulations with three different turbulence models and a DES simulation with the SST-DES model were performed in a industrial cyclone operating at high Reynolds number. The results in best agreement with the experiments were obtained with the Yakhot RNG LES turbulence model, followed by the Smagorinsky and the dynamic model. The results obtained with the SSTDES turbulence model diverged considerably from the experimental data, particularly in the lower sections (close to the cyclone bottom). This is probably due to the high aspect ratio used in these 
sections, which means that a much more refined grid in the axial direction would be necessary. The analysis of the average flow field suggest that the effect of turbulence modeling can be significant for particle separation.

\section{ACKNOWLEDGEMENTS}

The authors would like to tank FAPEMIG, CAPES and PETROBRAS for the financial support.

\section{REFERENCES}

Bernardo, S., 2005, Estudo dos Escoamentos Gasoso e Gás-Sólido em Ciclones pela Aplicação de Fluidodinâmica Computacional, Doctoral Thesis, Universidade Estadual de Campinas, SP. (in Portuguese)

Ferziger, J. H. and Peric, M., 2002, Computational Methods for Fluid Dynamics, Springer.

Germano, M., Piomelli, U., Moin, P. and Cabot, W. H., 1990, A Dynamic Subgrid-Scale Eddy Viscosity Model, American Institute of Physics, Vol. 7, pp. 1760-1795.

Gronald, G. and Derksen, J. J., 2010, Simulating Turbulent Swirling Flow in a Gas Cyclone: a Comparison of Various Modeling Approaches, Powder Technology, pp. 160-171.

Hoffman, A. C., Stein, L. E., 2008, Gas Cyclones and Swirl Tubes - Principles, Design and Operation, Second Edition, Springer - Verlag Berlin Heidelberg.

Lilly, D. K., 1992, A Proposed Modification of the Germano Subgrid-Scale Closure Method, Physics Fluids, Vol. 4, No. 3, pp. 633-635.

Menter, F. R., 1992, Improved Two Equation k$\omega$ Turbulence Models for Aerodynamic Flows, NASA Technical Memorandum 103975.

Narasimha, M., Brennan, M. and Holtham, P. N., 2006, Large Eddy Simulation of Hydrocyclone Prediction of Air-Core Diameter and Shape, International Journal of Mineral Processing, Vol. 80, pp. 1-14.

Narasimha, M., Brennan, M., Holtham, P. N. and Napier-Nunn, T. J., 2007, A Comprehensive CFD Model of Dense Medium Cyclone Performance, Minerals Engineering, Vol. 20, pp. 414-426.

Notay, Y., 2010, An Aggregation-Bases Algebraic Multigrid Method, Electronic Transactions on Numerical Analysis, Vol. 37, pp. 123-146.

Yakhot, A., Orsag, S. A., Yakhot, V. and Israeli, M., 1986, Renormalization Group Formulation of Large-Eddy Simulation, Journal Scientific Computing, Vol. 1, pp. 1-51.

Shalaby, H. H., 2007, On the Potential of Large Eddy Simulation to Simulate Cyclone Separators, Master Thesis, Von der Fakultät für Maschinebau der Technischen Universität Chemnitz, Germany.
Silveira-Neto, A., 2002, Turbulência nos Fluidos Aplicada, Faculdade de Engenharia Mecânica, Univesidade Federal de Uberlândia UFU, Uberlândia, MG. (in Portuguese)

Slack, M. D., Prasad, R. O., Bakker, A. and Boysan, F., 2000, Advances in Cyclone Modeling Using Unstructured Grids, Institution of Chemical Engineers, Vol. 78, Part A, pp. 1098-1104.

Strelets, M., 2001, Detached eddy Simulation of Massively Separated Flows, In $39^{\text {th }}$ AIAA Aerospace Sciences Meeting and Exhibit, American Institute of Aeronautics \& Astronautics, Reno, Nv.

Wegner, B., Maltsev, A., Schneider, C., Sadiki, A., Dreizler, A., and Janicka, J., 2004, Assessment of Unsteady RANS in Predicting Swirl Flow Instability Based on LES and Experiments, Heat and Fluid Flow, Vol. 25, pp. 528-536.

Received: June 20, 2012

Revised: July 20, 2012

Accepted: August 20, 2012 